\title{
Oxidation of hydrogen-passivated silicon surfaces by scanning near-field optical lithography using uncoated and aluminum-coated fiber probes
}

Madsen, Steen; Bozhevolnyi, Sergey I.; Birkelund, Karen; Müllenborn, Matthias; Hvam, Jørn Märcher; Grey, Francois

Published in:

Journal of Applied Physics

Link to article, DOI:

$10.1063 / 1.365847$

Publication date:

1997

Document Version

Publisher's PDF, also known as Version of record

Link back to DTU Orbit

Citation (APA):

Madsen, S., Bozhevolnyi, S. I., Birkelund, K., Müllenborn, M., Hvam, J. M., \& Grey, F. (1997). Oxidation of hydrogen-passivated silicon surfaces by scanning near-field optical lithography using uncoated and aluminumcoated fiber probes. Journal of Applied Physics, 82(1), 49-53. https://doi.org/10.1063/1.365847

\section{General rights}

Copyright and moral rights for the publications made accessible in the public portal are retained by the authors and/or other copyright owners and it is a condition of accessing publications that users recognise and abide by the legal requirements associated with these rights.

- Users may download and print one copy of any publication from the public portal for the purpose of private study or research.

- You may not further distribute the material or use it for any profit-making activity or commercial gain

- You may freely distribute the URL identifying the publication in the public portal 


\title{
Oxidation of hydrogen-passivated silicon surfaces by scanning near-field optical lithography using uncoated and aluminum-coated fiber probes
}

\author{
S. Madsen \\ DME-Danish Micro Engineering A/S, DK-2730 Herlev, Denmark Mikroelektronik Centret, Danmarks \\ Tekniske Universitet, DK-2800 Lyngby, Denmark \\ S. I. Bozhevolnyi \\ Institute of Physics, University of Aalborg, DK-9220 Aalborg, Denmark \\ K. Birkelund, M. Müllenborn, J. M. Hvam, and F. Grey \\ Mikroelektronik Centret, Danmarks Tekniske Universitet, DK-2800 Lyngby, Denmark
}

(Received 10 January 1997; accepted for publication 26 March 1997)

\begin{abstract}
Optically induced oxidation of hydrogen-passivated silicon surfaces using a scanning near-field optical microscope was achieved with both uncoated and aluminum-coated fiber probes. Line scans on amorphous silicon using uncoated fiber probes display a three-peak profile after etching in potassium hydroxide. Numerical simulations of the electromagnetic field around the probe-sample interaction region are used to explain the experimental observations. With an aluminum-coated fiber probe, lines of $35 \mathrm{~nm}$ in width were transferred into the amorphous silicon layer. (C) 1997 American Institute of Physics. [S0021-8979(97)00613-0]
\end{abstract}

\section{INTRODUCTION}

Nanolithography by scanning probe microscopy (SPM) on hydrogen-passivated silicon has become a very promising lithographic process. The technique relies on the local removal of hydrogen atoms, causing an immediate oxidation of the silicon surface in air. The oxide is used as an etch mask so that two-dimensional patterns can be transferred into the silicon. For lithographic purposes the process has been demonstrated using a scanning tunneling microscope (STM),${ }^{1-5}$ an atomic force microscope (AFM), ${ }^{6,7}$ and electron beam lithography. ${ }^{4,8}$ More recently, optically induced hydrogen desorption was reported. ${ }^{9-11}$

In this article we discuss results obtained using a scanning near-field optical microscope $(\mathrm{SNOM})^{12}$ to generate an oxide mask on an amorphous silicon $(a-\mathrm{Si})$ layer. Results for uncoated fiber probes are compared with those for aluminum-coated probes. Minimum power densities that are needed to achieve oxidation compare well with results obtained using a conventional far-field setup, suggesting that the dominant mechanism of SNOM induced oxidation is thermal desorption. Line scans with an uncoated fiber display a three-peak profile after etching the surface in potassium hydroxide $(\mathrm{KOH}) .{ }^{10}$ Numerical simulations based on a macroscopic self-consistent model ${ }^{13,14}$ have been used to explain the experimental observations. Taking the uncertainty of the precise shape of the probe into account, the model was found to be in good agreement with the observed silicon structures. Linewidths as small as $35 \mathrm{~nm}$ are produced using an aluminum-coated fiber probe with an aperture diameter of $\sim 100 \mathrm{~nm}$.

\section{OPTICAL NEAR-FIELD LITHOGRAPHY}

A schematic diagram of the SNOM apparatus is shown in Fig. $1 .{ }^{15}$ As in any other scanning probe configuration, the most important component is the probe, here a tapered single-mode optical fiber. For sample exposure, light from an argon ion laser operating at $457.9 \mathrm{~nm}$ is coupled into the probe. During the writing process, the laser output power is stabilized at $120 \mathrm{~mW}$.

Probes are fabricated using a combined heating/pulling process in a commercially available micropipette puller. ${ }^{16} \mathrm{~A}$ scanning electron microscope (SEM) is used to characterize the shape and size of the probe tip. Two SEM micrographs of an uncoated and an aluminum-coated probe are shown in Fig. 2. The taper angle of the uncoated probe, as measured by the SEM, is $18^{\circ}-20^{\circ}$. The diameter of the aperture of the aluminum-coated probe is $\sim 100 \mathrm{~nm}$. The thickness of the coating layer is $80-100 \mathrm{~nm}$. A shear-force microscope is applied to control the probe-to-sample distance, enabling imaging of the surface topography in a constant distance (damping) mode with a lateral resolution of $5-10 \mathrm{~nm} .{ }^{17}$

Samples for the writing process are layered silicon structures. A $\sim 25 \mathrm{~nm}$ thermal oxide is grown on a $\mathrm{Si}(100)$ substrate. A boron-doped $a$-Si layer with a thickness of $\sim 15 \mathrm{~nm}$ is then deposited onto the thermal oxide by dc magnetron sputtering. The samples used with aluminum-coated fiber probes were $\sim 55 \mathrm{~nm} a$-Si on $\sim 170 \mathrm{~nm}$ thermal oxide. For hydrogen passivation the samples are dipped in 5\% diluted hydrofluoric acid (HF) for $60 \mathrm{~s}$ and blown dry with $\mathrm{N}_{2}$. The passivation layer is suitable for lithographic processing after at least $4 \mathrm{~h}$ after HF dip. After light exposure the samples are etched in $28 \mathrm{wt} \% \mathrm{KOH}$ at room temperature for $35 \mathrm{~s}$, and characterized in an AFM operating in contact mode.

\section{A. Lithography with uncoated fiber probes}

The maximum far-field intensity emitted from an uncoated fiber probe is measured to be $\sim 25 \mathrm{~mW}$. With a scanning speed of $80 \mu \mathrm{m} / \mathrm{s}$, and assuming an effective illumination area of $1 \mu \mathrm{m}$ in diameter, the maximum illumination dose reaches $40 \mathrm{~kJ} / \mathrm{cm}^{2}$ which is sufficient for hydrogen desorption. ${ }^{9}$ Figure 3 shows an AFM image of two slightly displaced scan lines after the $\mathrm{KOH}$ etch. Each line scan has a profile consisting of three peaks, a narrow central peak, and 


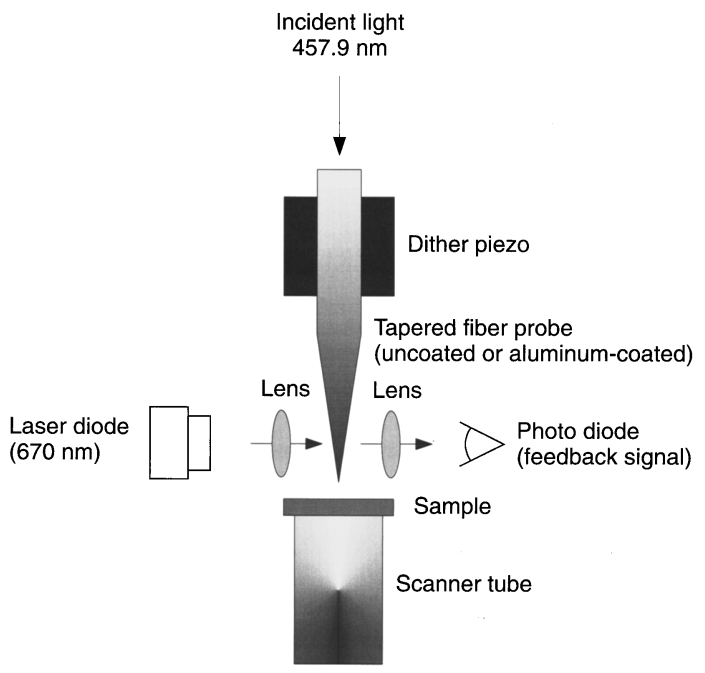

FIG. 1. Schematic diagram of the SNOM setup. The hydrogen-passivated silicon surface is illuminated through an optical fiber probe (uncoated or aluminum coated) at a wavelength of $457.9 \mathrm{~nm}$. A shear-force microscope is applied to control the probe-to-sample distance.

two slightly broader side peaks. Since the sample is illuminated through an uncoated fiber probe, we have previously proposed that the narrow central peak is due to the optical near-field emitted from the very end of the SNOM probe, whereas the broader side peaks are due to an optical interference pattern mainly dominated by the far-field penetrating the sidewalls of the uncoated probe. ${ }^{10}$ The full width at halfmaximum of the central peak is $\sim 115 \mathrm{~nm}$. The typical line-
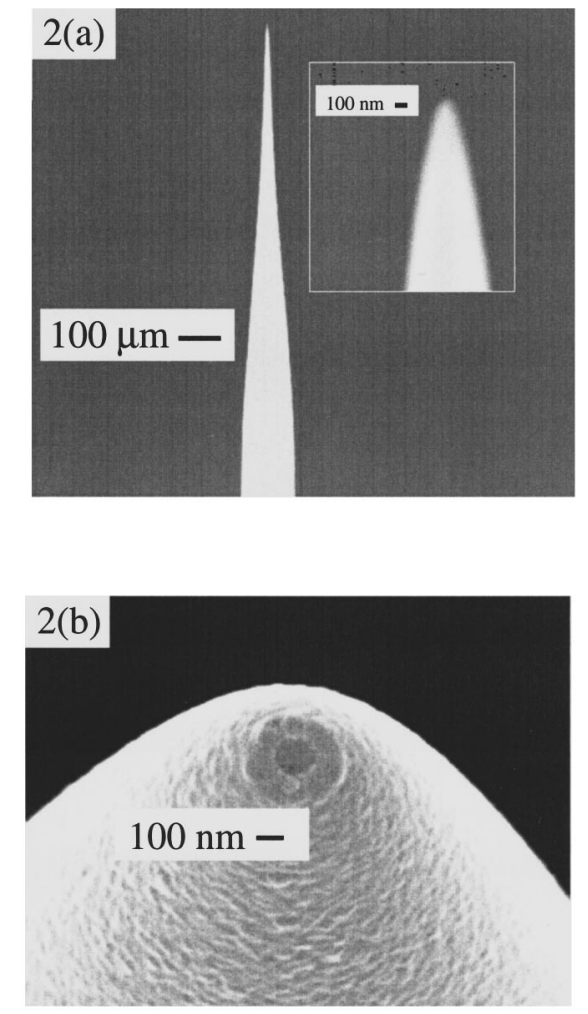

FIG. 2. SEM micrographs of an uncoated and an aluminum-coated fiber probe.

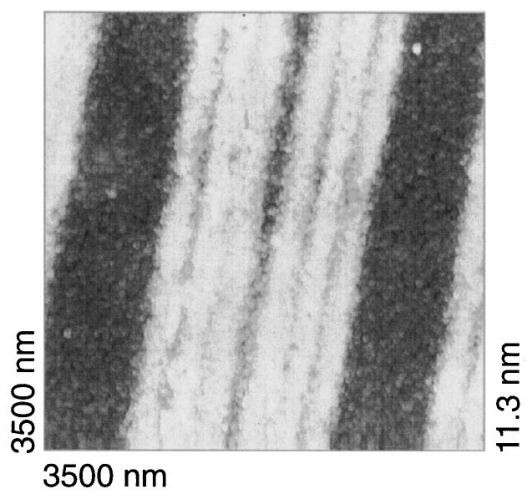

FIG. 3. AFM image after the KOH etch of two slightly displaced scan lines. The narrow central peak is believed to be written with the optical near field, whereas the slightly broader side peaks stems from an optical interference pattern dominated by the far field emitted through the sidewalls of the uncoated fiber probe. The linewidth of the central peak is $\sim 115 \mathrm{~nm}$. Typical linewidths of the side peaks are $215-225 \mathrm{~nm}$. The average peak-to-peak distance, between the central peak and the side peaks is $245 \mathrm{~nm}$.

width of the side peaks is $215-225 \mathrm{~nm}$, and the average peak-to-peak distance between the central peak and the side peaks is $245 \mathrm{~nm}$.

To test our interpretation of the experimental observations, the field distribution at the sample surface was modeled using a macroscopic self-consistent two-dimensional model for the reflection SNOM. ${ }^{13,14}$ The system under consideration is shown in Fig. 4, where the three regions (probe, air gap, and sample) are assumed to be three homogeneous dielectric media. The modeling is carried out for TE waves, i.e., the electric field is perpendicular to the figure plane.

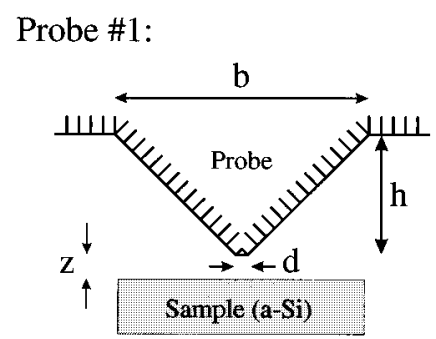

Probe \#2:

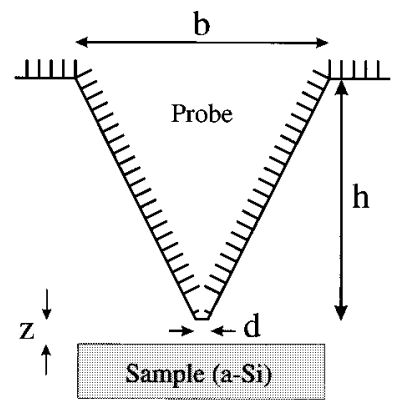

FIG. 4. Geometry of the probe-sample system considered in the numerical simulations. Probe No. 1: $b=2 \mu \mathrm{m}, h=1 \mu \mathrm{m}$, and $d=20 \mathrm{~nm}$. Probe No. 2 : $b=2 \mu \mathrm{m}, h=2 \mu \mathrm{m}$, and $d=20 \mathrm{~nm}$. The illumination wavelength is 457.9 $\mathrm{nm}$. The refractive index of the sample is estimated to be 4.35 . The probeto-sample distance is $5 \mathrm{~nm}$, i.e., $z=5 \mathrm{~nm}$. 


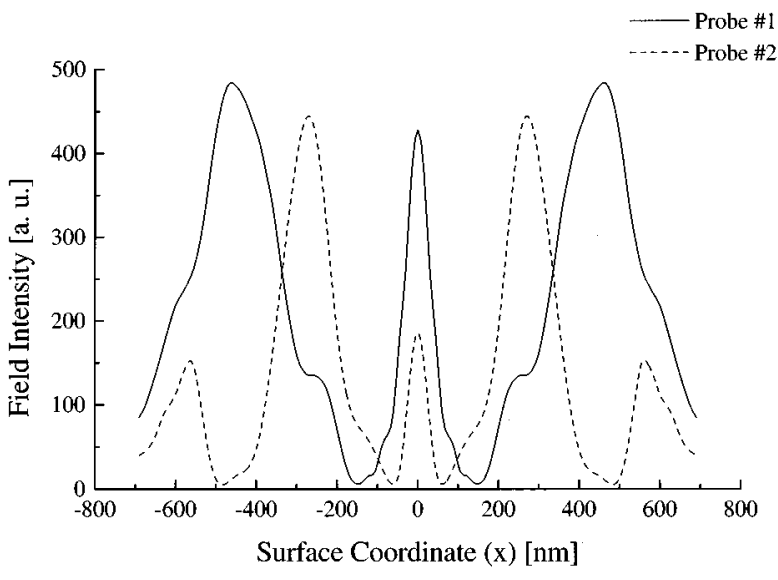

FIG. 5. Simulated field intensity at the sample surface for two different probe shapes: probe No. 1 (solid line) and probe No. 2 (dashed line).

The results of the numerical calculations are shown in Fig. 5 for two different probe shapes. Probe No. 1 has a taper angle of $45^{\circ}$, whereas probe No. 2 has a taper angle of $26.6^{\circ}$, which is similar to that of the fabricated uncoated probes in Fig. 2(a). The end facets of both probes are $20 \mathrm{~nm}$. The three-peak intensity profile is observed in the simulations for both probes. The peak spacing depends on the shape of the probe, with the smallest taper angle yielding the smallest distance between the central peak and the side peaks. Also, the intensity of the individual peaks depends on the probe shape.

It should be kept in mind that the intensity distributions presented in Fig. 5 are two dimensional. Qualitatively, they can be viewed as radial cuts through the three-dimensional radiation pattern, assuming rotation symmetry around the fiber axis. To compare the calculated results with experiments, it is necessary to convolute the full intensity pattern with a straight line that represents the scanning of the probe, and then take a cross-sectional profile of the resulting intensity distribution.

Assuming that the calculated intensity profiles are symmetric with rotation around the fiber axis, the sample exposure can thus be calculated along a scanning line according to the following relation:

$$
E(x)=\frac{1}{v} \int_{-\infty}^{\infty} I\left(\sqrt{x^{2}+y^{2}}\right) d y,
$$

where $v$ is the scanning speed and $x$ and $y$ are the surface coordinates. The scanning direction is along the surface coordinate $y$. The result of the calculations for probe No. 2 is shown in Fig. 6 (solid line). The widths of the central and side peaks are 93 and $261 \mathrm{~nm}$, respectively. The peak-topeak distances between the central peak and the side peaks are $237 \mathrm{~nm}$. For comparison a typical cross-sectional profile of Fig. 3 is also shown in Fig. 6 (dashed line). As can be seen, the simulation is in good quantitative agreement with the experimental observations. The calculated exposure distribution of the three peaks, however, differs significantly from the measured profiles. In principle, detailed modeling of the tip shape used in the calculation may lead to better agreement. We note, though, that the oxidation and subse-

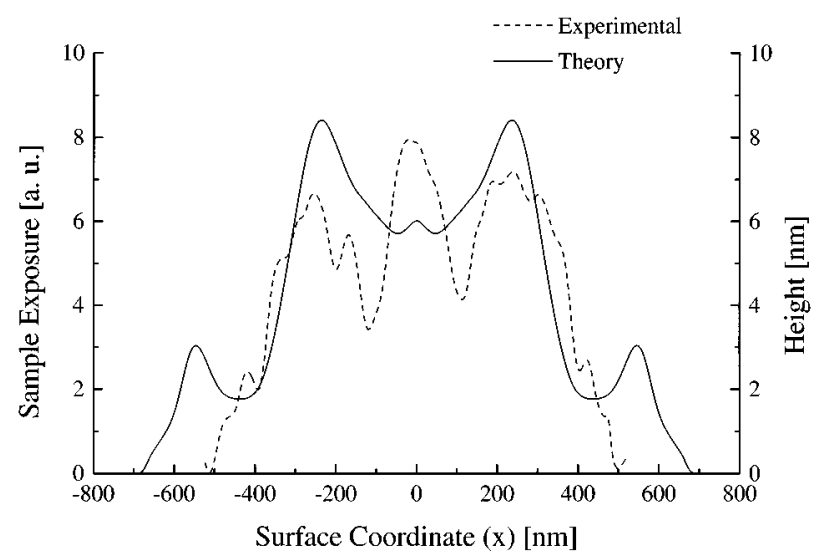

FIG. 6. Simulated sample exposure along a scanning line for probe No. 2 (solid line). The widths of the central peak and the side peaks are 93 and 261 $\mathrm{nm}$, respectively. The peak-to-peak distance between the central peak and the side peaks is $237 \mathrm{~nm}$. A typical cross-sectional profile from Fig. 3 is also shown (dashed line).

quent etching steps used to make the measured profiles are not yet understood in detail, and may have a strong nonlinear dependence on exposure, making detailed modeling a questionable exercise at this stage.

\section{B. Lithography with aluminum-coated fiber probes}

Given that the side peaks for uncoated probes are due to light escaping from the shank of the probe, aluminum-coated fiber probes should be capable of increased resolution due to the better confinement of the light source.

In order to get a significant improvement in resolution, aperture diameters smaller than $200 \mathrm{~nm}$ are required. This however, raises another problem: the typical output intensity of a metalized fiber probe is considerably lower than the uncoated probe because the input intensity must be kept below a value capable of evaporating the coating. The output intensity of an aluminum-coated probe, measured in the far field as a function of the input intensity, is shown in Fig. 7. The measured output intensity goes linear with the input intensity until the input reaches $4.5 \mathrm{~mW}$. At this point the aluminum coating starts to evaporate from the probe causing the output intensity to increase dramatically. To demonstrate

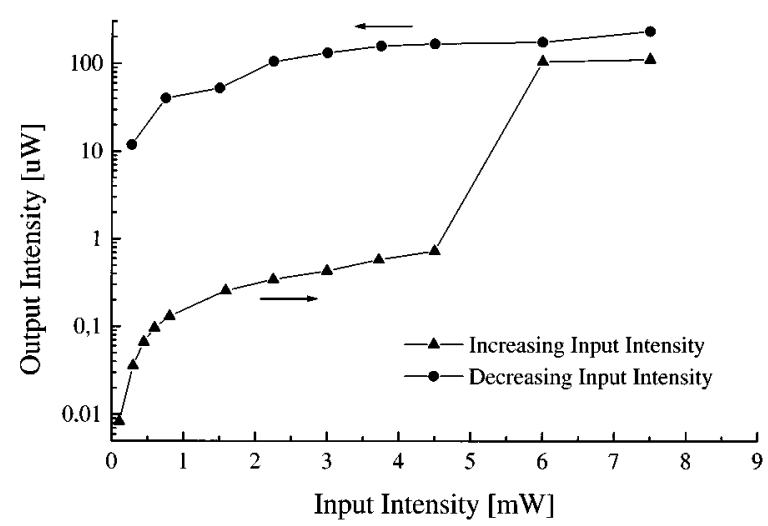

FIG. 7. Measured output power as a function of input power for an aluminum-coated fiber probe with an aperture diameter of $\sim 100 \mathrm{~nm}$. 


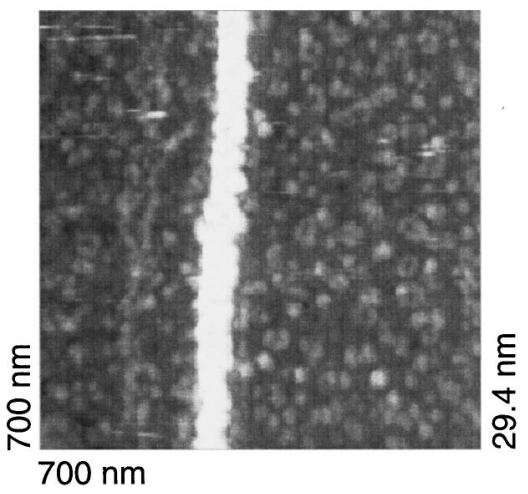

FIG. 8. AFM image after the $\mathrm{KOH}$ etch of single line written via sample exposure through an aluminum-coated fiber probe with an aperture diameter of $100 \mathrm{~nm}$. The illumination wavelength is $457.9 \mathrm{~nm}$. The power density applied to the sample was estimated to be $130 \mathrm{~kW} / \mathrm{cm}^{2}$.

that the coating is really damaged, the output was also measured with decreasing input intensity, revealing a clear hysteresislike effect.

For maximum sample exposure the input power was adjusted to $4.5 \mathrm{~mW}$, which corresponds to an output power of $\sim 0.7 \mu \mathrm{W}$. The near-field intensity cannot be deduced directly from the measured far-field intensity. However, assuming a point dipole radiation through an aperture of 100 $\mathrm{nm}$ in diameter placed $50 \mathrm{~nm}$ from the dipole center, the near-field intensity at the sample surface can be calculated to be $130 \mathrm{~kW} / \mathrm{cm}^{2}$. In this estimation the far-field intensity of the dipole radiation (in a half-space) equals the measured output intensity of the aluminum-coated probe, i.e., $0.7 \mu \mathrm{W}$. With a scanning speed of $54 \mu \mathrm{m} / \mathrm{s}$ the exposure dose is calculated to be $241 \mathrm{~J} / \mathrm{cm}^{2}$, which is over two orders of magnitude less than the exposure dose applied with the uncoated probe. For this reason a different type of sample was chosen. To maximize local heating of the $a$-Si layer due to illumination, and hence the oxidation rate, increasing the thickness of the underlying oxide layer is found to be beneficial, presumably due to better thermal isolation of the $a$-Si layer. ${ }^{18}$ Here an $\sim 170 \mathrm{~nm}$ oxide was used compared to the $\sim 25 \mathrm{~nm}$ oxide used in the experiments with uncoated probes.

An AFM image of a single line written with an aluminum-coated fiber is shown in Fig. 8. The total scan area is $700 \times 700 \mathrm{~nm}^{2}$. A cross-sectional profile of the line is shown in Fig. 9. The effect of the coating is evident, in that the far-field induced side peaks have disappeared and the width of the central peak is reduced from 115 to only $54 \mathrm{~nm}$. De-convoluting the shape of the AFM tip, the actual linewidth may be as narrow as $35 \mu \mathrm{m}$. Also, we do not observe any oxidation when the light to the probe is switched off, in contrast to unexpected oxidation effects that have been reported previously with uncoated fiber probes under no illumination. ${ }^{10}$

\section{COMPARING NEAR-FIELD AND FAR-FIELD RESULTS}

It is of interest to compare the conditions for near-field oxidation with those already reported for far-field oxidation using a focused laser beam in a laser direct-write system. ${ }^{19}$

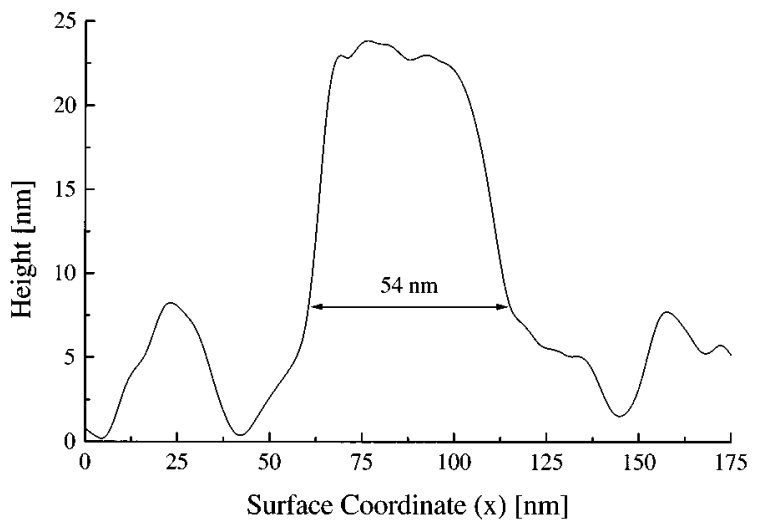

FIG. 9. Cross-sectional profile of the single line in Fig. 8. The apparenet width of the line is $54 \mathrm{~nm}$. The real linewidth may be as narrow as $35 \mu \mathrm{m}$.

The required power density for the formation of an oxide layer sufficient to resist $\mathrm{KOH}$ etching is shown in Fig. 10 as a function of exposure time (dashed line). For high speed scanning (short exposure times), the minimum power density remains constant for over three orders of magnitude. In this regime the required power density reaches $200 \mathrm{~kW} / \mathrm{cm}^{2}$ which is sufficient to heat the $a$-Si layer to temperatures of about $700 \mathrm{~K} .{ }^{19}$ Such temperatures can cause thermal desorption of the hydrogen passivation layer. Above $100 \mathrm{~ms}$ the minimum power density drops nearly linearly with exposure time. This change in slope suggests that an additional mechanism contributes to the hydrogen desorption process in the low power density regime. Possible mechanisms are direct optical desorption or desorption by optically induced excess carriers. It should be noted that the far-field experiments were carried out on samples identical to those used for the uncoated SNOM probes.

In the SNOM case with uncoated fiber probes four intensity levels were used for sample exposure $(0.9,2.5,9.0$, and $25 \mathrm{~mW}$ ). Assuming that the diameter of the exposed area is $1 \mu \mathrm{m}$, power densities ranging from $115 \mathrm{~kW} / \mathrm{cm}^{2}$ to $3.2 \mathrm{MW} / \mathrm{cm}^{2}$ were applied to the sample. A fixed scanning speed of $80 \mu \mathrm{m} / \mathrm{s}$ yields a maximum exposure time of 12.5 $\mathrm{ms}$. For the highest power densities $(0.32,1.15$, and $3.2 \mathrm{MW} / \mathrm{cm}^{2}$ ), which, according to Fig. 10, are in the region

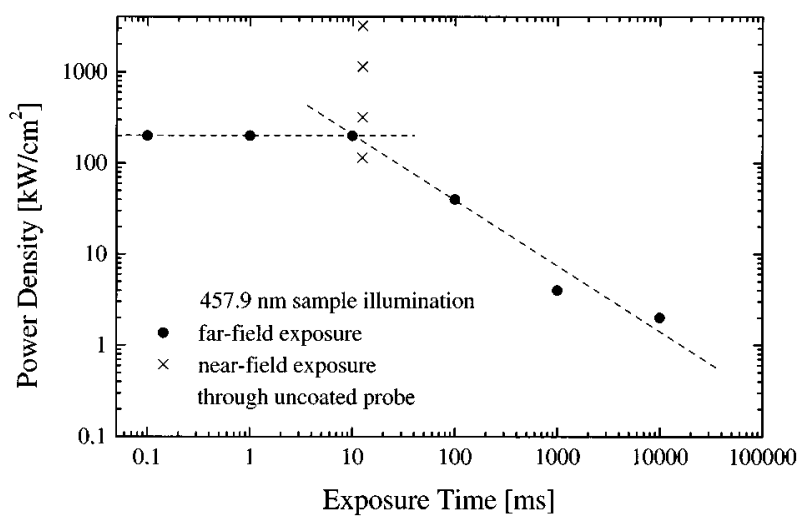

FIG. 10. Minimum power density, at $\lambda=457.9 \mathrm{~nm}$, required for the formation of an oxide layer sufficient for $\mathrm{KOH}$ etching (dashed line). The four crosses indicate the working points for SNOM with uncoated fiber probes. 
of thermal desorption, the oxide mask is sufficient for selective etching in $\mathrm{KOH}$. At the lowest power density of $115 \mathrm{~kW} / \mathrm{cm}^{2}$, the selectivity becomes significantly weaker and no features are observed after the KOH etch. This observation agrees well with the data for far-field exposure presented in Fig. 10.

For the aluminum-coated probes, we estimate the power density to be $130 \mathrm{~kW} / \mathrm{cm}^{2}$. Given the scanning speed of 54 $\mu \mathrm{m} / \mathrm{s}$, this result would fall below the threshold measured by far-field techniques. At lower power densities of $87 \mathrm{~kW} / \mathrm{cm}^{2}$, the written features begins to be less clearly defined, and by further reducing the power density to $43 \mathrm{~kW} / \mathrm{cm}^{2}$ no features are observed after ecthing in $\mathrm{KOH}$. As discussed above, the likely origin of this discrepancy is the greater oxide thickness used for the experiments with coated tips, which effectively increases the local heating achievable at a given power density. Since the experiments with aluminum-coated probes are not directly comparable to other results, we have not included these results in Fig. 10.

\section{CONCLUSIONS}

We have shown that both uncoated and aluminumcoated fiber probes in a reflection SNOM configuration can be used for local depassivation of hydrogen-passivated silicon. The generated oxide is used as an etch mask to transfer two-dimensional nanostructures into an $a$-Si layer. Linewidths as small as $35 \mathrm{~nm}$ are obtained after the $\mathrm{KOH}$ etch using coated fiber probes to illuminate the sample. Sample exposure through uncoated fiber probes result in a three-peak line profile along the scanning direction. This profile has been modeled semiquantitatively using a macroscopic selfconsistent model, confirming that the two side peaks in the observed profile are due to light escaping from the shank of the probe.

\section{ACKNOWLEDGMENTS}

The authors would like to thank Niels C. R. Holme (RISØ National Laboratory, Roskilde, Denmark) for stimulating discussions during the fabrication of aluminum-coated fiber probes. This work was supported by the Danish Academy of Technical Sciences.

${ }^{1}$ J. A. Dagata, J. Schneir, H. H. Haray, C. J. Evans, M. T. Postek, and J. Bennet, Appl. Phys. Lett. 58, 2001 (1990).

${ }^{2}$ J. W. Lyding, T. C. Shen, J. S. Hubacek, J. R. Tucker, and G. C. Abeln, Appl. Phys. Lett. 64, 2010 (1994).

${ }^{3}$ H. Sugimura and N. Nakagiri, Appl. Phys. Lett. 66, 1430 (1995).

${ }^{4}$ N. Kramer, J. Jorritsma, H. Birk, and C. Schönenberger, J. Vac. Sci. Technol. B 13, 805 (1995).

${ }^{5}$ N. Kramer, H. Birk, J. Jorritsma, and C. Schönenberger, Appl. Phys. Lett. 66, 1325 (1995).

${ }^{6}$ H. C. Day and D. R. Allee, Appl. Phys. Lett. 62, 2691 (1993).

${ }^{7}$ E. S. Snow and P. M. Campbell, Appl. Phys. Lett. 64, 1932 (1994).

${ }^{8}$ K. Tsubouchi and K. Masu, Thin Solid Films 228, 312 (1993).

${ }^{9}$ N. Kramer, H. Niesten, and C. Schönenberger, Appl. Phys. Lett. 67, 2989 (1995).

${ }^{10}$ S. Madsen, M. Müllenborn, K. Birkelund, and F. Grey, Appl. Phys. Lett. 69, 544 (1996).

${ }^{11}$ K. Birkelund, M. Müllenborn, S. Madsen and F. Grey, Superlattices Microstruct. 20, 555 (1996).

${ }^{12}$ D. W. Pohl, W. Denk, and M. Lanz, Appl. Phys. Lett. 44, 651 (1984).

${ }^{13}$ S. Berntsen, S. I. Bozhevolnyi, and E. A. Bozhevolnaya, J. Opt. Soc. Am. A 10, 878 (1993).

${ }^{14}$ S. I. Bozhevolnyi, S. Berntsen, and E. A. Bozhevolnaya, J. Opt. Soc. Am. A 11, 609 (1994).

${ }^{15}$ DME-Rasterscope SNOM, Danish Micro Engineering A/S, Herlev, Denmark.

${ }^{16}$ Model P-2000 laser based micropipette puller, Sutter Instrument Company, CA.

${ }^{17}$ E. Betzig, P. L. Finn, and J. S. Weiner, Appl. Phys. Lett. 60, 2484 (1992).

${ }^{18} \mathrm{~K}$. Birkelund, S. Madsen, M. Mullenborn, and F. Grey (unpublished).

${ }^{19}$ M. Müllenborn, K. Birkelund, S. Madsen, and F. Grey, Appl. Phys. Lett. 69, 3013 (1996). 